\section{PSMA-Targeted Therapeutics: A Tale About Law} and Economics

TO THE EDITOR: Without any doubt, the breakthrough in radiopharmaceuticals targeting prostate-specific membrane antigen (PSMA) has stirred up nuclear medicine and radiopharmaceutical research and ultimately boosted industrial engagement in the entire field. The first-in-humans application of the ${ }^{68} \mathrm{Ga}$-labeled PET tracer ${ }^{68}$ Ga-PSMA-11 in 2011 (hence its present name; initially referred to as ${ }^{68}$ Ga-HBED-PSMA) (1) galvanized the community and led to a fierce race toward the matching ${ }^{177} \mathrm{Lu}$-therapeutics. To the best of our knowledge, this games' first goal was scored by ${ }^{177}$ Lu-PSMA I\&T, being the first radiometal-based PSMA ligand that was successfully applied for therapy of metastatic castration-resistant prostate carcinoma (2). Shortly thereafter, one novel compound after another appeared, aiming primarily at therapeutic applications (e.g., ${ }^{177}$ Lu-PSMA-617) (3) and purely imaging applications (e.g., ${ }^{18}$ F-PSMA-1001) (4). Interestingly, the commercial interest also led to unexpected short squeezes in some supply chains. For example, PSMA-617 became widely unavailable one fine day as a result of a change in ownership of the patent rights, leaving many clinical radiopharmacies at odds on how to settle their respective requirements.

At this point, it is interesting to note that no patents were filed for PSMA-11 and PSMA I\&T, most likely because the tremendous commercial success of this class of radiopharmaceuticals was not foreseeable at that time. As a result, PSMA-11 was widely used and became a global de facto standard for PSMA PET in an incredibly short time. Because it is sold by numerous manufacturers worldwide and is available as a labeling kit, it is still one of the most frequently used PSMA tracers, despite other agents that might arguably be more potent. In principle, the same used to be true for PSMA I\&T. For quite a time, many departments were relying on it for their clinical routine. However, the market situation for this compound also took an interesting turn when a fairly old chelator patent entered the stage.

PSMA I\&T features a particular flavor of DOTA as the chelator moiety (5), namely, a tetraazacyclododecane with 3 acetic acids and 1 glutaric acid side arm-hence its acronym DOTAGA (6). This bifunctional chelator structure was developed more than $20 \mathrm{y}$ ago by Helmut Mäcke et al., and a patent application was filed for it on May 11, 2001 (7). The patent slumbered for quite a while but eventually was licensed from Basel University by CheMatech, a company that codeveloped and marketed DOTAGA anhydride (8), a valuable building block for making DOTAGAfunctionalized compounds. By February 2019, parts of the same patent, covering the use of DOTAGA in all its conjugates, allegedly were sublicensed to another player in the field in order to gain property rights for PSMA I\&T (9). As a countermove, others have announced that any applicable license fees for PSMA I\&T, made from a stock of DOTAGA anhydride purchased from CheMatech before February 2019, have inherently been settled by originally purchasing the DOTAGA used for making PSMA I\&T from a licensee (i.e., CheMatech), and thus, the PSMA I\&T produced therewith may nonetheless be purchased and used without infringing any law (10).

Whether this is true shall not be commented on here, since this legal skirmish just became history anyway. It remains to be asked to what extent the expiry of the DOTAGA patent (by May 11, 2021) bears the potential to change the game of thrones that is being played in the field of PSMA theranostics. The fact that a powerful therapeutic PSMA radioligand, ${ }^{177}$ Lu-PSMA I\&T, may be manufactured and used without any patent restrictions from May 11 onward will probably have an impact on future development, availability, and pricing of similar agents. To all researchers in the field, this tale might be a lesson — and a warning - to secure their intellectual property in sufficient time to save themselves from late regrets.

\section{REFERENCES}

1. Eder M, Schäfer M, Bauder-Wüst U, et al. ${ }^{68} \mathrm{Ga}$-complex lipophilicity and the targeting property of a urea-based PSMA inhibitor for PET imaging. Bioconjug Chem. 2012;23:688-697.

2. Weineisen M, Schottelius M, Šimeček J, et al. ${ }^{68} \mathrm{Ga}$ - and ${ }^{177}$ Lu-labeled PSMA I\&T: optimization of a PSMA-targeted theranostic concept and first proof-of-concept human studies. J Nucl Med. 2015;56:1169-1176.

3. Benešová M, Bauder-Wüst U, Schäfer M, et al. Linker modification strategies to control the prostate-specific membrane antigen (PSMA)-targeting and pharmacokinetic properties of DOTA-conjugated PSMA inhibitors. J Med Chem. 2016;59:17611775 .

4. Cardinale J, Schäfer M, Benešová M, et al. Preclinical evaluation of ${ }^{18}$ F-PSMA-1007, a new prostate-specific membrane antigen ligand for prostate cancer imaging. $\mathrm{J} \mathrm{Nucl}$ Med. 2017;58:425-431.

5. Weineisen M, Šimeček J, Schottelius M, Schwaiger M, Wester HJ. Synthesis and preclinical evaluation of DOTAGA-conjugated PSMA ligands for functional imaging and endoradiotherapy of prostate cancer. EJNMMI Res. 2014;4:63.

6. Eisenwiener KP, Powell P, Mäcke HR. A convenient synthesis of novel bifunctional prochelators for coupling to bioactive peptides for radiometal labelling. Bioorg Med Chem Lett. 2000;10:2133-2135.

7. Maecke H, Eisenwiener K, Powell P, inventors; University Hospital Basel, assignee. Prochelators of radiometal labeled molecules. European patent EP1289571 (B1). July 21, 2004.

8. Bernhard C, Moreau M, Lhenry D, et al. DOTAGA-anhydride: a valuable building block for the preparation of DOTA-like chelating agents. Chemistry. 2012;18: 7834-7841.

9. PSMA I\&T. ATT Scintomics website. https://sci-att.com/psma-it-2/. Accessed August 23, 2021.

10. PSMA. ABX Advanced Biochemical Compounds website. https://www.abx.de/ Information/Index/?viewId=Pro_tracer. Accessed August 23, 2021.

\author{
Johannes Notni \\ Technische Universität München \\ München, Germany \\ E-mail: johannes.notni@tum.de
}

Published online March 31, 2021.

DOI: $10.2967 /$ jnumed.121.262308 